\title{
Combined Analysis of Systematic and Random Uncertainties for Different Noise-Figure Characterization Methodologies
}

\author{
A. Collado, J. M. Collantes, L. De la Fuente ${ }^{1}$, N. Otegi, L. Perea, M. Sayed ${ }^{2}$ \\ Electricity and Electronic Department, University of the Basque Country, Bilbao, Spain \\ ${ }^{1}$ DICOM, University of Cantabria, Santander, Spain \\ ${ }^{2}$ Agilent Technologies, Santa Rosa, CA
}

\begin{abstract}
This paper presents a simulation tool for the rigorous analysis of the final uncertainty associated to different methodologies for noise figure characterization. The simulation tool permits the analysis of the combined effect of systematic errors and underlying uncertainties versus any significant characteristic of the DUT or measurement setup. Some application examples are presented showing the suitability of the proposed approach to determine the most efficient characterization methodology for a given DUT and measurement setup.
\end{abstract}

\section{INTRODUCTION}

Accurate noise figure characterization is a key issue in the development of circuits and devices for demanding wireless applications. Significant efforts are being made in order to improve measurement accuracy by selecting the most suitable methodology that minimizes and/or corrects the various sources of errors [1-2]. The basic formulations associated to these methodologies ( $Y$ factor, cold source) are often complemented with additional correction terms in order to eliminate errors arising from systematic effects, such as mismatch related effects, noise added by the receiver, etc. In general, these corrections require additional measurements or calibrations, such as Sparameter or noise-parameter characterization.

However, uncertainty affecting the noise-figure measurement has diverse origins. On the one hand, the correction terms used to remove systematic errors have their own uncertainty due to the incomplete knowledge of the required value of the correction. On the other hand, some other systematic errors may not be taken into account by the expressions used for noise figure calculation. In addition there is also uncertainty arising from random effects (such us connector variability, jitter...), uncertainty related with the limited accuracy of the measurement instruments, with the imperfect knowledge of the hot and cold noise temperatures, etc. The suitability of a particular methodology, having a specific level of corrections, is a function of the DUT and setup characteristics. In some cases, complicating the measurement process to include correction terms will not necessarily lead to more accurate results, while in other cases corrections may be crucial for achieving the desired accuracy. Circuit manufacturers need to determine, for given characteristics of DUT and measurement setup, which methodology for noise figure calculation is able to provide the required accuracy without spending unnecessary time and effort in extra measurements and corrections.

In order to provide reliable answers to the mentioned issues, a rigorous and realistic simulation tool that enables the joint analysis of random and systematic uncertainties in a noise-figure characterization process, is presented in this work. As proposed by Randa for the case of noiseparameter uncertainty calculation [3], a Monte Carlo method is used here to simulate the combined effect of the uncertainties of the underlying quantities on the final noise-figure uncertainty.

The bases of the proposed simulation tool are described in the following section. Some representative examples are discussed in section III. Finally some conclusions are summarized.

\section{SIMULATION TOOL}

\section{A. Modeling of the Measurement Setup}

The generic measurement setup of Fig. 1 has been modeled. This setup includes a noise source characterized by its cold and hot temperatures $(T c, T h)$ and by their respective reflection coefficients $\left(\Gamma s_{-} c, \Gamma s_{-} h\right)$. Two 2ports devices (input and output), defined by their $S$ parameters, model any passive block connected at the input and output ports of the Device Under Test (DUT). The noise receiver is characterized by its input reflection coefficient ( $\Gamma r e c)$, its gain-bandwidth product (BGrec) and its four noise parameters (Fmin, Rn, $\Gamma o p t)$. Linearity and thermal drift of the receiver are also modeled through its $1 \mathrm{~dB}$ compression point $(P I d B)$ and a thermal drift factor (Dth) respectively. Finally, the DUT is defined by its $S$ parameters and by its four noise parameters (Fmin', $R n$ ', Гopt'). All these parameters are the "true" values of the DUT and measurement setup and they are used to 
simulate the actual noise power measured by the setup. The simulated noise powers are considered as the "true" measured noise power values.

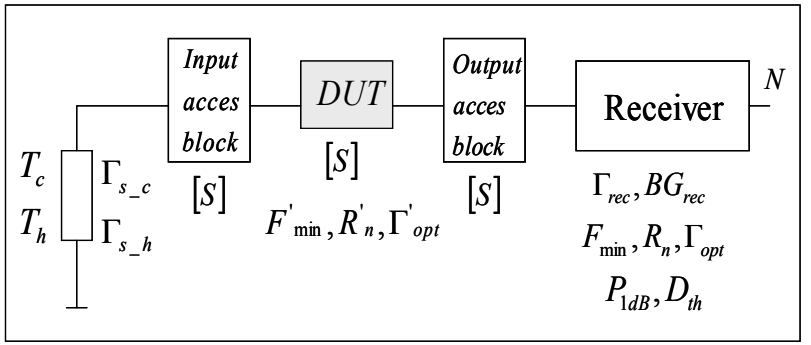

Fig. 1. Modeling of the measurement setup.

On the other hand, the "true" value of the DUT noise figure Ftrue for a particular source termination (defined by the user) is calculated from their noise parameters. Ftrue is used as the reference value in the analysis.

\section{B. Basic Methodologies and Corrections for Errors Arising from Systematic Effects}

Two basic formulations for the calculation of noise figure are considered in this analysis: the well-known $Y$ factor technique, used by the most common commercially available instruments for noise-figure measurement, and the cold-source technique. The bases of these two techniques are well reported in the literature $[1,2,4,5]$. Only a short summary is given here.

The Y-factor technique, in its simplest version, only requires scalar power measurements and relies on the good match of both DUT and measurement setup. The noise figure of the DUT is computed from two noise powers $(N h, N c)$ measured with the noise source at its hot and cold temperatures $(T h, T c)$ respectively. Assuming that the cold temperature $T c$ is equal to the reference temperature $T_{0}=290 \mathrm{~K}$, the noise figure can be obtained from (1), which represents the simplest formulation for noise figure calculation.

$$
F=\left(\frac{T_{h}}{T_{0}}-1\right) /\left(\frac{N_{h}}{N_{c}}-1\right)
$$

The cold-source technique computes the noise figure from a single noise measurement $(N c)$ with a 50 -Ohm source impedance, at room temperature, connected to the input of the DUT. For that, the gain-bandwidth product of the receiver (BGrec) and the available gain of the DUT (Gav) needs to be previously determined. The gainbandwidth product is typically obtained from a calibration in which the noise source is directly connected to the receiver and the noise is measured for the two temperatures (hot and cold). The DUT available gain is calculated from $S$-parameter measurements. Again, assuming that the room temperature is equal to the reference temperature $T_{0}$, the simplest formulation for cold source is:

$$
F=\frac{N_{c}}{G_{a v} \cdot T_{0} K B G_{r e c}}
$$

The basic Y-factor and cold-source formulations represented by (1) and (2) can be complemented with the addition of correction terms in order to eliminate errors arising from systematic effects, such as mismatch related effects, noise added by the receiver, etc. The most significant corrections that are taking into account by the proposed simulation tool are the following:

- De-embedding of the DUT noise figure from the whole system noise figure (cascade of DUT and receiver) by making use of the Friis formula for cascaded stages.

- Calculation of the DUT available gain required for a rigorous application of the Friis formula. Classical $Y$ factor based approaches compute the DUT insertion gain from scalar power measurements. Both gains are similar for perfect match but they can diverge significantly when DUT output match degrades.

- Vector corrections accounting for any access network that may be placed at the input and output ports of the DUT.

- Differences between the cold temperature $(T c)$ and the reference temperature $\left(T_{0}=290 \mathrm{~K}\right)$

- Variations in the reflection coefficient of the noise source from cold to hot temperatures $\left(\Gamma s_{-} c \neq \Gamma s_{-} h\right)$. Obviously, this effect cannot be completely removed from the $Y$-factor formulation, since that would require the knowledge of the DUT noise parameters, which are $a$ priori unknown.

- Dependence of the receiver noise figure on the source termination through the set of four noise parameters (Fmin, Rn, Гopt).

\section{Uncertainty Analysis}

Independent uncertainties can be associated to any of the parameters from the set of "true" values characterizing the measurement setup, to the $S$-parameters of the DUT and to the "true" measured noise power values. The estimated uncertainty of each parameter (only B-type uncertainties in this analysis [6]) is introduced as a standard deviation. The real and imaginary parts of the complex parameters are generated independently. A new set of simulated data is generated by randomly choosing values from a Gaussian distribution centered at the "true" value of each parameter. The possibility of taking into 
account correlations in the measurements has also been included in the simulation tool, since their presence can have significant impact on the final uncertainty [3]. From the set of new parameter values the noise figure can be calculated using any methodology, ranging from the simplest $Y$-factor and/or cold-source formulations to their complete set of corrections mentioned above. In the standard manner of Monte Carlo methods, this process is repeated a large amount of times (over 1000). At each iteration the individual error is calculated as $e(d B)=$ $a b s(F c a l c(d B)-F t r u e(d B))$. Then, the mean, $m_{e}$, and the standard deviation of the error, $\sigma_{e}$, are computed. The final uncertainty of the noise figure, is calculated as $u=\sigma_{e}$ $+m_{e}$.

\section{APPLICATION EXAMPLES}

Uncertainty in a noise figure characterization process strongly depends on DUT and receiver characteristics and on the selected methodology. Consequently, the aim of the following examples is not to extract general results on noise figure uncertainty, but to demonstrate the advantages of associating a rigorous uncertainty analysis to the noise figure characterization.

The considered DUT for the following examples is a 44 $\mathrm{GHz}$ amplifier for radio astronomy applications. Its main characteristics are: $S_{21}=10 \mathrm{~dB} \angle 7.9^{\circ}, S_{11}=0.48 \angle 102^{\circ}, S_{22}$ $=0.41 \angle 43^{\circ}$ and $F \approx 3 \mathrm{~dB}$. A commercially available wave-guide noise source, with Excess Noise Ratio (ENR) $=10.1 \mathrm{~dB}$ at $44 \mathrm{GHz}$, is utilized. The reflection coefficients at this frequency for both cold and hot temperatures are: $\Gamma s \_c=0.15 \angle-20^{\circ}$ and $\Gamma s \_h=0.08 \angle 4^{\circ}$. In fact, the actual noise figure of the DUT for $\Gamma_{s} c=$ $0.15 \angle-20^{\circ}$ will be considered as the reference value, Ftrue, in the analyses. The receiver is a custom built noise meter for Q-band noise figure measurements, with the following parameters at $44 \mathrm{GHz}$ : Fmin $=6.7 \mathrm{~dB}, R n=60$ $\Omega \Gamma o p t=0.08 \angle 271^{\circ}, \Gamma r e c=0.085 \angle-63^{\circ}$.

First, three different versions of the $Y$-factor technique, each one having different corrections, will be analyzed versus DUT input match. YFstd corresponds to the standard $Y$-factor procedure, in which only scalar power measurements are used. YF1 adds to YFstd a correction term to account for variations in the reflection coefficient of the noise source $\left(\Gamma s_{-} c \neq \Gamma s_{-} h\right)$. YF2 adds to YF1 two additional corrections: the use of the DUT available gain in the Friis formula and the dependence of the receiver noise figure on the source termination. In this first example no uncertainty levels are added to the variables involved in the calculation, so the obtained errors exclusively arise from systematic effects.

Results of the analysis are plotted in Fig. 2. As expected, Fig. 2 shows that the error associated to YFstd increases as input match worsens. In addition, for this particular case, the most critical correction concerns the changes in the noise source reflection coefficient, while little improvement is gained with the rest of correction terms. This could be expected too, because the noise source presents a considerable variation in its reflection coefficient from hot to cold states, at $44 \mathrm{GHz}$.

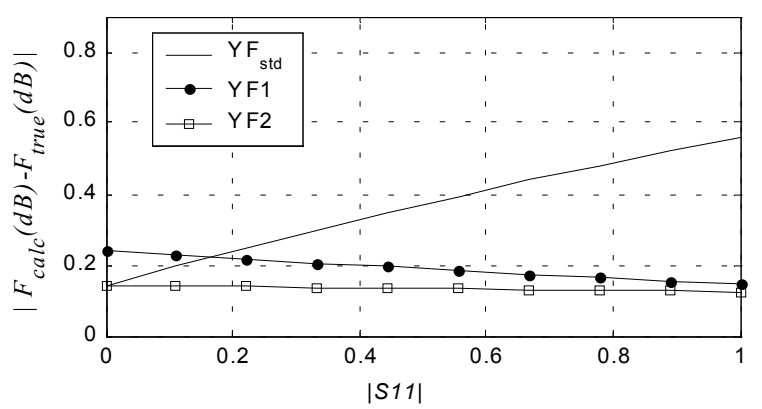

Fig. 2. Comparison of three versions of the Y-factor technique. Systematic error versus DUT input match

However, results can be different when uncertainties are incorporated to the variables in the simulation. Type B uncertainties are estimated for the proposed DUT and measurement setup. Typical uncertainty values, given by manufacturer's specifications at Q band, are assumed for $E N R$ and for every variable associated to scattering measurements. An uncorrelated 2\% uncertainty, representing the jitter in the measurement, is considered for the noise power measurements. In addition, $2 \%$ uncertainty is also applied to the receiver noise parameters.

When these uncertainties are applied, the three methodologies under study provide more comparable results (Fig. 3). However, it is important to remark that the uncertainty corresponding to YF2 ( $Y$ factor with full corrections) is slightly higher than the one associated to $Y F 1$ (only $\Gamma s_{-} c \neq \Gamma s_{-} h$ correction), confirming that, in some cases, the addition of correction terms that are not actually required can become an additional source of uncertainty.

Results from Figs. 2 and 3 have already confirmed that changes in the noise source reflection coefficient are critical in our particular example. Therefore two additional methodologies that could be convenient for a case like this will be analyzed next. The first one consists of including an isolator at the output of the noise source, while using the standard $Y$-factor technique, YFstd. The $S$ parameters of the isolator have been measured and vector corrections are applied to account for the isolator effect on the noise source excess noise. The second one is the coldsource technique $(C S)$. This technique can be suitable in 
this case because it avoids the connection of noise source at its hot temperature to the DUT. The cold source analyzed in this example includes all the corrections for systematic errors except for the dependence of the receiver noise figure on the source termination.

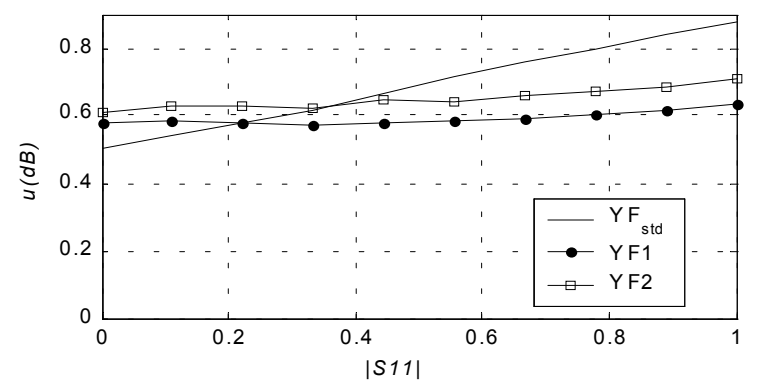

Fig. 3. Uncertainty comparison of three versions of the $Y$ factor technique when uncertainties in the variables are applied.

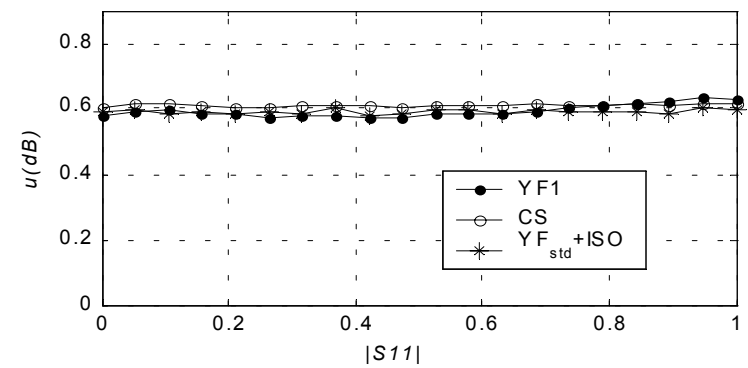

Fig. 4. Uncertainty comparison of cold-source and Y-factor based methodologies versus DUT input match.

Results from this analysis are shown in Fig. 4, where the previous $Y F 1$ has also been plotted for comparison. The three methodologies provide comparable results versus DUT input match. This is a reasonable result since the three of them tend to minimize (or avoid) the critical changes in the noise source reflection coefficient.

The previous analysis could now be repeated versus any other parameter involved in the calculation. As an example, Fig. 5 shows the same analysis performed versus DUT gain (with input match $S_{11}$ fixed to $0.48 \angle 100^{\circ}$ ). Two significant effects have to be outlined from Fig. 5. First, as expected, uncertainty gets amplified as DUT gain lowers. Second, for high gain devices, the uncertainty associated to the $Y$-factor based methodologies augment substantially. This increase is due to the non-linear behavior of the receiver at high power levels that was modeled by its $1 \mathrm{~dB}$ compression point. Y-factor based methodologies can be more critically affected by receiver compression because of the $10 \mathrm{~dB}$ extra noise added by the noise source at $T h$.

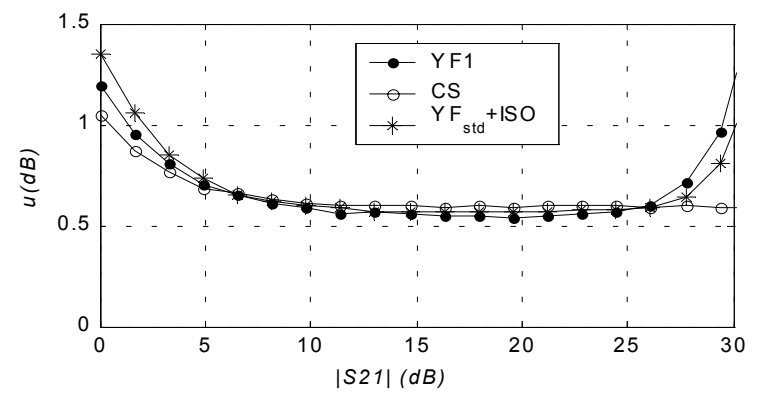

Fig. 5. Uncertainty comparison of cold-source and Y-factor based methodologies versus DUT gain.

\section{CONCLUSION}

A simulation tool for computing the final uncertainty associated to different noise figure characterization methodologies, has been presented. It allows the combination of systematic and random errors in the analysis. Some representative examples have shown the suitability of such a tool to help circuit developers to choose the most convenient methodology that guarantees the required accuracy for given DUT and setup characteristics.

\section{ACKNOWLEDGEMENT}

The authors wish to acknowledge the University of the Basque Country for partially supporting this work.

\section{REFERENCES}

[1] D. Vondran, "Noise Figure Measurement: Corrections related to match and Gain," Microwave Journal, March 1999, pp. 22-38.

[2] R. Meierer, C. Tsironis, "An On-Wafer Noise Parameter Measurement Technique with Automatic Receiver Calibration, ", Microwave Journal, March 1995, pp. 22-37.

[3] J. Randa, "Simulations of noise-parameter uncertainties," 2001 IEEE MTT-S Int. Microwave Symp. Dig., Seattle, June 2001, pp. 1845-1848

[4] V. Adamian, A. Uhlir, "A Novel Procedure for Receiver Noise Characterization, " IEEE Trans. on Instruments \& measurements, Vol. 22, No. 2, June 1973, pp.181-182.

[5] "Fundamentals of RF and Microwave Noise Figure measurements, " Agilent Application Note 57-1

[6] B. N. Taylor, C. E. Kuyatt "Guidelines for Evaluating and Expressing the Uncertainty of NIST Measurement Results," NIST Tech. Note 1297, 1994 